\title{
Low-temperature Plasma Jet Activated Media Induces Apoptosis in Bladder Cancer Cells via the Cytochrome c/caspase 3 Pathway
}

Hideo Fukuhara ( $\sim$ fukuhara@kochi-u.ac.jp)

Kochi Medical School

Endre J. Szili

Future Industries Institute

Jun-Seok Oh

Osaka City University

Kawada Chiaki

Kochi Medical School

Shinkuro Yamamoto

Kochi Medical School

Atsushi Kurabayashi

Kochi Medical School

Mutsuo Furihata

Kochi Medical School

Masayuki Tsuda

Kochi Medical School

Hiroshi Furuta

Kochi University of Technology

Howard D. Lindsay

Lancaster University

Robert D. Short

Lancaster University

Akimitsu Hatta

Kochi University of Technology

Keiji Inoue

Kochi Medical School

\section{Research Article}

Keywords: plasma jet, reactive oxygen species (ROS), bladder cancer cells, plasma activated media (PAM) 
Posted Date: July 19th, 2021

DOl: https://doi.org/10.21203/rs.3.rs-701484/v1

License: (c) (i) This work is licensed under a Creative Commons Attribution 4.0 International License. Read Full License 


\section{Abstract}

Current methods used to treat non-muscle invasive bladder cancer are inadequate due to a high recurrence rate after surgery and occurrence of adverse events such as interstitial pneumonia following intravesical instillation therapy. Low-temperature plasma is a new form of physical therapy that provides a rich source of reactive oxygen species (ROS). Oxidative solutions, created by pre-treatment of aqueous media with plasma before application to target cells, lead to the destruction of cancer cells through oxidative stress pathways. This study focuses on the effects of plasma activated media (PAM) in bladder cancer cells. PAM treatment increases up regulation of p21 and down regulation of Cyclin D and CdK4 leading to cell cycle arrest, and concomitantly depolarises the mitochondrial membrane leading to increased mitochondrial ROS production. Cell cycle arrest and increased mitochondrial ROS production induce apoptosis in bladder cancer cells in vitro and in a bladder cancer tumour in vivo via the proapoptotic caspase $3 /$ cytochrome c pathway. These observations highlight the potential of plasma activated solutions as a new adjuvant therapy in the clinical treatment of bladder cancer.

\section{Introduction}

Low-temperature plasma technology is a potentially new form of physical therapy to combat cancers. Unlike other physical cancer therapies including radiotherapy, plasma is unique as it enables concomitant treatment from a complex mix of molecules such as reactive oxygen species (ROS) and reactive nitrogen species (RNS) as well as other physical components including electromagnetic radiation and heat [1]. Another interesting property of plasma is that unlike X-ray irradiation, plasma is non-ionising which makes the treatment less destructive to surrounding healthy cells and tissue. Numerous studies have demonstrated the advantageous unique properties of plasma by showing how the technology can be applied to induce death in cancer cells without detrimental damage to non-diseased cells as summarised in the review by Ratovitski et al [2]. In addition to direct plasma treatment of cancer cells, plasma can also be indirectly used to treat cancer by pre-treating media with plasma before application to cells; this is often referred to as plasma activated media therapy [3-7].

Possible reasons why plasma technology can potentially be used for targeted cancer therapy rely on the unique phenotypic and behavioural characteristics of cancer cells. Some of these factors include cancer cells generally having a higher number of aquaporin channels and altered levels of certain lipid types and cholesterol in such a way that these features reduce the barrier effects of the cell membrane to ROS [812]. These features allow cancer cells to easily uptake plasma generated ROS in certain circumstances, which makes these cells more susceptible to oxidative damage by the plasma treatment [13-15]. Plasma induced oxidative stress may also disrupt biomolecular binding events to inhibit proliferative signalling pathways in cancer cells [16]. In addition, the higher proliferative nature of many cancer cells also increases their sensitivity to plasma treatment. The reason for this is that cancer cells are usually under an increased oxidative stress condition due to their higher metabolic activity that increases their vulnerability to external ROS insults. Proliferative cancer cells also spend a greater percentage of the time 
in S-phase of the cell cycle (i.e. DNA synthesis stage), which makes the new DNA more susceptible to oxidative damage [17].

Despite the widespread interest in the use of plasma technology for cancer treatments, only a few published studies have focussed on the application of the technology to bladder cancers. However, bladder cancer remains a major healthcare challenge. There are more than 70,000 and 150,000 new bladder cancer diagnoses each year in the United States and Europe, respectively $[18,19]$. The standard treatment for non-muscle invasive bladder cancer is transurethral resection of the bladder tumour (TURBT) [20]. TURBT is a physical form of therapy involving the use of a specialised thin, rigid tool called a resectoscope, which is placed into the bladder via the urethra. The resectoscope enables visualisation of the internal bladder whilst removing tumour tissue with a heated wire loop attached to the device or with a laser directed through the device. TURBT can be used to remove the tumour with only minimal short-term side effects. However, the incidence of tumour recurrence is very high, and repeated TURBT treatments lead to significant scarring of the bladder tissue and eventually loss of bladder function. Multicentric tumour development, incomplete killing of residual bladder cancer cells by intravesical chemotherapy post TURBT, and untreated residual lesions that are endoscopically invisible during surgery, including flat lesions such as dysplasia and carcinoma in situ (CIS), are common factors of tumour recurrence.

There is opportunity provided by plasma technology to develop new treatment approaches to improve bladder cancer patient outcomes, particularly with respect to decreasing the frequency of tumour recurrence. The potential of plasma in treating bladder cancer cells was demonstrated in an in vitro study by Joh et al who showed the high efficacy of a plasma jet, operated with helium and an admixture of oxygen (to increase ROS production), at eradicating human bladder cancer (EJ) cells [21]. The plasma treatment induced apoptosis (cell death) in the cells through increased oxidative stress, which is in line with the background theory of how plasma works in cancer therapy (as described above). Another in vitro study was reported by Mohades et a/ who investigated the use of a helium plasma jet for the treatment of human bladder SCaBER cells [22]. Two treatment methods were compared: (1) direct plasma exposure of the cells, and (2) indirect plasma exposure with the cell culture media that was pre-treated with plasma before application to cells (referred to as plasma activated media or PAM). Both methods of treatment were found to be equally as effective at eradicating the bladder cancer cells. Cell death was shown to occur through apoptosis linked to the caspase 3 signalling pathway. Keidar et al showed that the in vitro results could be translated to an in vivo subcutaneous human bladder tumour (SCaBER) mouse model [23]. Reduction in tumour growth achieved by helium plasma jet treatment was linked to changes in expression of several genes associated with the apoptotic and oxidative stress pathways.

In this study, we aim to provide further insights into the mechanisms of how PAM deactivates bladder cancer cells. The cell media used in this study serves as a pseudo physiological-like solution to study how PAM influences the function of bladder cancer cells both in vitro and in vivo. Herein, we focus on how the oxidative stress conditions produced by the plasma treatment induces apoptosis in the bladder cancer cells via the caspase 3/cytochrome c pathway. We hypothesise that this can occur through two 
processes, either by causing cell cycle arrest or via increased mitochondrial ROS production as indicated below:

Hypothesis 1: PAM à Oxidative stress à Mitochondrial ROS generation à Cytochrome c release à Caspase 3 activation à Apoptosis

Hypothesis 2: PAM à Oxidative stress à Cell cycle arrest (GO/G1 phase) à Cytochrome c release à Caspase 3 activation à Apoptosis

\section{Materials And Methods \\ 2.1 Production of PAM}

PAM was produced with an atmospheric-pressure low-temperature He plasma jet configured with an external single electrode on a $150 \mathrm{~mm}$ long glass capillary with an internal diameter of $2.4 \mathrm{~mm}$ (Figure 1a) [24-27]. Power was supplied by a high-voltage bipolar square wave pulse of $7 \mathrm{kV}$ (peak-to-peak) at a frequency of $10 \mathrm{kHz}$. He gas was fed into the glass capillary at a fixed gas flow rate of 2 SLM. PAM was generated by plasma jet treating $3 \mathrm{~mL}$ of Dulbecco's Modified Eagle Medium (DMEM) (ThermoFisher SCIENTIFIC) in a $60 \mathrm{~mm}$ polystyrene tissue culture dish at a $10 \mathrm{~mm}$ distance from the liquid surface for 5 , 10 and 15 minutes. Comparative level of ROS generated in the PAM was measured by adding $10 \mu \mathrm{L}$ of $100 \mathrm{mM}$ luminol (Nacalai tesque) to $3 \mathrm{~mL}$ PAM, incubating for 1 minute at ambient temperature and then imaging using an IVIS spectrum 200 system (Xenogen) with 1 minute exposure. Fluorescence intensity of the images was analysed using Livinglmage 3.0 software.

\subsection{Human bladder cell lines and cell culture conditions}

The human bladder carcinoma cell line 253JB-V and human urothelial cancer (UC) cell line T24 were maintained in DMEM supplemented with $10 \% \mathrm{v} / \mathrm{v}$ fetal bovine serum (FBS, Life Technologies Inc) and $200 \mu \mathrm{g} / \mathrm{ml}$ streptomycin. Cells were cultured at $37^{\circ} \mathrm{C}$ in a humidified atmosphere containing $5 \% \mathrm{CO}_{2}$.

\subsection{PAM treatment of cells}

A density of $5 \times 10^{3}$ cells/well was seeded into a 96-well and cultured for 24 hours in $100 \mu \mathrm{L}$ of media. The media was then removed and replaced with $100 \mu \mathrm{L}$ of PAM. Cells were incubated in PAM and cultured for 3 hours and/or 24 hours before assessment as detailed in the following sections. Control group represents cells not exposed to PAM but otherwise treated identically compared to the PAM treated cells.

\subsection{Cell viability assay}


A volume of $10 \mu \mathrm{L}$ of $5 \mathrm{mg} / \mathrm{ml}$ 3-(4, 5-dimethyl-thiogol-2-yl)-2, 5-diplenyltetrazollium (MTT, Sigma) was added directly into each well and incubated for 3 hours to allow the mitochondria dehydrogenase to convert MTT into an insoluble formazan product. The media was aspirated, and the sediment dissolved in dimethyl sulfoxide (DMSO, FUJIFILM Wako Pure Chemical Corporation). Absorbance was measured at $570 \mathrm{~nm}$ on a SpectraMax 190 microplate reader (Molecular Devices) to determine the cell viability.

\subsection{Flow cytometry analysis of cell death, mitochondrial membrane potential and mitochondrial ROS, and cell cycle}

Cells were trypsinised by $2.5 \%$ trypsin (ThermoFisher SCIENTIFIC) and washed with phosphate buffered saline (PBS, Sigma). Cells were then centrifuged for 5 minutes and stained as detailed in the following protocols. To identify each cell cycle phase a BrdU flow cytometer kit (BD Biosciences) was used according to the manufacture's protocol. Identification of apoptotic and necrotic cells was achieved by double staining of $5 \mu \mathrm{L}$ annexin V-APC (BD Pharmingen) and $5 \mu \mathrm{L}$ propidium iodide (PI, Sigma) dyes for 10 minutes. Cells were twice washed with binding buffer and then measured. Mitochondria membrane potential $(\Delta \Psi \mathrm{m})$ and mitochondrial ROS were analysed by staining with $250 \mathrm{nM}$ tetramethylrhodamine methyl ester (TMRE, Takara Bio) and $5 \mu \mathrm{M}$ MitoSox (ThermoFisher SCIENTIFIC) for 15 minutes to determine mitochondria membrane potential $(\Delta \Psi \mathrm{m})$ and reactive oxygen species (ROS) in mitochondria, respectively. The stained cells for each method were processed for LSRFortessa or FACScan (BD Biosciences). A minimum 10,000 cells per sample was acquired and subsequently analysed with Flow Jo software. The $\Delta \Psi_{\mathrm{m}}$ and mitochondrial ROS were measured as fluorescence intensity in the histogram, respectively. Cell death was determined from the total percentage of cells stained positive for annexin $\mathrm{V}$ and PI in the flow cytometry graphs. In the flow cytometry graph, the sections in the lower left, lower right and upper right correspond to intact, apoptotic and necrotic cells, respectively.

\subsection{Quantification of intracellular cytochrome c and caspase 3}

Intracellular concentration of cytochrome $\mathrm{c}$ and caspase 3 were measured by first trypsinising cells and lysing with cell lysis buffer 2 (R\&D systems). Cytochrome c concentration was measured using a commercially available kit (R\&D systems, catalogue no. DCTCO) according to the manufacturer's protocol. The cell lysates were mixed with cytochrome c conjugate for 2 hours at room temperature. After $2 \mathrm{~h}$, the wells were washed and incubated with substrate solution for 30 minutes. The absorbance of each well was measured at $570 \mathrm{~nm}$ using a Molecular Devices Thermomax microplate reader. For determination of the intracellular caspase 3 concentration, the cell lysates were mixed with reaction buffer containing $20 \mathrm{mM}$ 4-(2-hydroxyethyl)-1-piperazineethanesulfonic acid (HEPES) buffer at pH 7.5, $0.1 \mathrm{M} \mathrm{NaCl}$ and $10 \mu \mathrm{M}$ of N-Acetyl-Asp-Glu-Val-Asp-7-amido-4-Methylcoumarin as a caspase 3 reporter molecule. Caspase 3 cleaves the reporter molecule resulting in release of 7-amino-4-methylcoumarin 
(AMC), which was measured using a fluorescence spectrophotometer at $570 \mathrm{~nm}$ on a Molecular Devices Thermomax microplate reader.

\subsection{Western blot analysis of cell cycle regulatory factors}

Following 24 hours after PAM treatment, cells were harvested and lysed with lysis buffer and protease inhibitors. Lysate protein was boiled with sample buffer for 5 minutes and separated by SDS-PAGE on a $15 \%$ polyacrylamidegel. Afterwards the proteins were transferred electrophoretically onto polyvinylidenedifluoride membranes. Non-specific binding site were blocked with PBS containing $1 \%$ bovine serum albumin (BSA). After blocking, the membranes were probed with appropriate antibodies against cyclin D, cdk4 and p21. All antibodies were purchased from Santa Cruz Biotechnology Inc. Antibody detection was performed using an Enhanced Chemiluminescence Assay (Pierce Biotechnology, Rockford, IL, USA).

\subsection{PAM treatment of in vivo subcutaneous bladder cancer tumour and analysis of treatment outcome}

Seven-week-old female BALB/c nu/nu mice were housed in plastic cages with stainless steel grid tops in an air-conditioned room with a 12 hour light-dark cycle maintained at room temperature and provided with water and food ad libitum in the institute for animal experiments of Kochi Medical School. Animal experiments were conducted in accordance with the institutional guidelines and regulations and reviewed by the animal experiment and welfare committee of Kochi Medical School. The animal experimental protocol was approved by the Institutional Animal Care and Use Committee of Kochi University Permit Number; J-16, L-6 and M-27. A total 10 mice were used in this study. The study was carried out in compliance with the ARRIVE guidelines.

Each mouse was injected in the dorsal region subcutaneously with $2 \times 10^{6} 253 \mathrm{JB}-\mathrm{V}$ cells suspended in $100 \mu \mathrm{L}$ of DMEM. Tumour was grown to a diameter of approximately $105 \mathrm{~mm}^{3}$, the tumour-bearing mice were randomly divided into two groups (control and treatment group). Dorsal skin of tumour was inverted and exposed to PAM for 30 minutes. A total of 6 mice and 4 mice were analysed for the PAM treatment group and untreated control group, respectively.

Following 24 hours after treatment, all mice were euthanised by the injection of pentobarbital, and the tumours were removed for pathological analysis. The tumour volume was calculated using the following formula: $\mathrm{V}=\mathrm{A} \times \mathrm{B}$, where $\mathrm{A}$ and $\mathrm{B}$ are orthogonal diameters of the tumours that were measured using $\mathrm{a}$ caliper. Harvested tumours were fixed in $20 \%$ formalin at room temperature for 48 hours. Each tumour was processed into paraffin and cut into $10 \mu \mathrm{m}$ thick sections. The resected tumour sections were stained using the terminal deoxynucleotidyl transferase dUTP nick end labelling (TUNEL) method, anti-Ki-67 antibody and with hematoxylin and eosin (H\&E). 
The TUNEL assay was used to measure the volume of the degenerated lesion within the tumour mass. The degenerated area was calculated according to the following formula: $V=C \times D$, where $C$ and $D$ are orthogonal diameters of the degenerated lesion measured using a microscope. The TUNEL-positive apoptotic cells were counted through a high-power field lens. More than 1000 tumour cells were counted to calculate the apoptosis index (Al) from these areas. Apoptotic cells were not evaluated from the vicinity of necrotic areas.

\subsection{Statistical analysis}

Statistical analysis was performed using JMP 8 software (SAS Institute Japan, Tokyo, Japan). Student-t tests were used to compare the data. Statistical significance was defined by a $p$ value of $<0.05$.

\section{Results And Discussion}

\subsection{Production of PAM for targeted bladder cancer treatment}

PAM was prepared by treating DMEM with a helium plasma jet as shown in Figure $1 \mathrm{a}$. Figure $1 \mathrm{~b}$ shows typical voltage and current waveforms during operation of the plasma jet. Figure $1 \mathrm{c}$ shows related optical emission spectrum of the plasma, respectively. Prominent emission peaks are observed for the $\mathrm{OH}^{\star}$ radical at $308 \mathrm{~nm}$ and exited $\mathrm{O}^{*}$ at $777 \mathrm{~nm}$ indicative of high energy components produced by the plasma in the air that subsequently produce ROS through downstream reactions in the liquid phase [25, 28]. In addition, the UV emitted from the plasma can generate ROS in situ through photolysis [29]. Measured and simulated spectrum of the $\mathrm{N}_{2}$ second positive system between $290 \mathrm{~nm}$ and $410 \mathrm{~nm}$ indicated the gas temperature to be near ambient temperature. The level of ROS produced in the media can be easily controlled by the plasma treatment time. A plasma treatment of 15 minutes produced a statistically significant increase in the relative level of ROS in the media (Figure 1d). PAM was shown to reduce the metabolic activity of two bladder cancer cell types 235JB-V and T24 at 3 hours post treatment (Figure 1 e). The metabolic activity of both bladder cancer cell types did not recover for at least 24 hours (Figure 1e). Macroscopic assessment of the cell morphology reveals marked changes post treatment for both cells (Figure 1f). Both bladder cell types generally exhibit a typical epithelial-like, polygonal morphology as seen in the micrographs taken for these cells. At 3 hours post treatment, most of the 235JB-V cells are round, indicating cell shrinkage a morphological characteristic of apoptosis, whereas the shape of the T24 cells was largely unaffected. At 24 hours, the majority of 235JB-V and T24 are round in morphology, which is indicating that both cells may be undergoing apoptosis at this timepoint.

\subsection{PAM initiated apoptosis in bladder cancer cells}


To assess if the reduced metabolic activity is associated with increased cell death, both 253JB-V and T24 cells post treatment were labelled with annexin V-APC for apoptosis and propidium iodide (PI) for all dead cells. Double staining cells with annexin V-APC and PI can be used to distinguish between apoptotic and necrotic cells by flow cytometry. Untreated cells at the 24 hours incubation timepoint and PAM treated cells at 3 hours and 24 hours post treatment were analysed. In the flow cytometry graphs PI cells are seen at the left-hand side of the second quadrant (Q2), V-APC cells in the upper left of Q3 and unstained (live) cells in bottom right of Q4 (Figures 2a-f). As seen in Figures 2a and 2d, the majority of 253JB-V and T24 cells (93.9\% and 95\%, respectively) before PAM treatment were annexin V-APC and PI negative, indicating that most of these cells are alive. At 3 hours post treatment, an increase in the number of apoptotic and necrotic cells was observed (Figures $2 \mathrm{~b}$ and $2 \mathrm{e}$ ). At 24 hours post treatment, $22.5 \%$ and $28.0 \%$ of 253JB-V cells and $24.3 \%$ and $26.3 \%$ of T24 cells, were apoptotic and necrotic, respectively (Figures $2 \mathrm{c}$ and $2 \mathrm{f}$ ). From Figure 1e, at 24 hours post treatment, we expect the number of non-viable cells in the whole cell population to be $46.8 \%$ and $46.9 \%$ for $253 \mathrm{JB}-\mathrm{V}$ and T24 cells, respectively. Therefore, out of the nonviable cell population at 24 hours post treatment, the percentage of apoptotic cells equates to $55.6 \%$ and $52.0 \%$ for the 253JB-V and T24 cells, respectively. This data shows that the PAM treatment readily induces apoptosis in both bladder cancer cell lines. To confirm this, we measured the concentration of caspase 3 in the bladder cancer cells. Caspase 3 is a frequently activated protease involved with dismantling the cell during the formation of apoptotic bodies [30]. Data for caspase 3 is not available at the 3 hours post treatment timepoint. However, at 24 hours post treatment, $1.7 \mathrm{ng} / \mathrm{mL}$ and $1.5 \mathrm{ng} / \mathrm{mL}$ of caspase 3 was detected in the 253JB-V and T24 cells, which was above the concentrations detected in the untreated cells (Figure $2 \mathrm{~g}$ ). In addition, the concentration of cytochrome $\mathrm{c}$ was measured in the bladder cancer cells. Cytochrome $\mathrm{c}$ is a mitochondrial protein that is released into the cytosol to initiate apoptosis when a cell receives an apoptotic signal [31]. Figure $2 \mathrm{~h}$ shows that the cytochrome $\mathrm{c}$ concentration at 24 hours post treatment in the 253JB-V and T24 cells, increased to $160.1 \mathrm{ng} / \mathrm{mL}$ and $168.4 \mathrm{ng} / \mathrm{mL}$, respectively, as compared to the untreated cells. The steady-state concentrations of cytochrome $\mathrm{c}$ in the untreated 253JB-V and T24 cells was also relatively high at $148.5 \mathrm{ng} / \mathrm{mL}$ and 121.9 $\mathrm{ng} / \mathrm{mL}$, respectively. This is expected because cytochrome $\mathrm{c}$ also has a major function in the mitochondria in adenosine triphosphate (ATP) synthesis [31] to supply energy to cells, and the proliferative nature of the bladder cancer cells increase their energy (ATP) requirements.

\subsection{Role of mitochondria in PAM induced apoptosis in bladder cancer cells}

We next tested our first hypothesis that PAM treatment induces apoptosis in bladder cancer cells by upregulation of mitochondrial ROS production. For these experiments, the 253JB-V and T24 cells were stained with tetramethylrhodamine methyl ester (TMRM) and MitoSox to determine mitochondria membrane potential $\left(\Delta \Psi_{\mathrm{m}}\right)$ and mitochondrial ROS, respectively. A high $\Delta \Psi_{\mathrm{m}}$ is often linked to elevated levels of mitochondrial ROS and is an important indicator of the cell's health [32,33]. During states of high $\Delta \Psi \mathrm{m}$, the charge within the cell is less negative resulting in cell depolarisation. The percentage of 
depolarised cells was analysed by flow cytometry and is shown Figure 2i. PAM treatment induced a sustained increase in the percentage of depolarised cells with $26.7 \%$ of $253 \mathrm{JB}-\mathrm{V}$ cells and $47.6 \%$ of T24 cells depolarised at 24 hours post treatment. The relative level of ROS in the mitochondria detected by flow cytometry is shown in Figure 2j. Cell deploarisation was accompanied by an increase in mitochondrial ROS production compared to the untreated cells with up to a 1.63-fold and 4.97-fold increase at 24 hours post treatment in the 253JB-V and T24 cells, respectively. Overall, these results indicate that cell depolarisation and increased mitochondrial ROS is linked to the induction of apoptosis in bladder cancer cells by the PAM treatment. However, the results show that the mitochondrial changes are more pronounced in the T24 cells compared to the 253JB-V cells, which may be attributed to differences in the molecular characteristics between the cells [34].

\subsection{Role of cell cycle arrest in PAM induced apoptosis in bladder cancer cells}

We next tested our second hypothesis if PAM induces apoptosis in bladder cancer cells by arresting the cell cycle. Changes in the cell cycle can regulate the apoptotic response [35]. Cell cycle progression was monitored by labelling cells with Bromodeoxyuridine (BrdU). BrdU is a thymidine analog that is inserted into DNA during cell cycling and hence is used to identify proliferating cells. The results from the flow cytometry analysis of the BrdU stained cells are presented in Figures 3a-d. Figures $3 a$ and $3 b$ shows the percentage of 253JB-V and T24 cells in the sub-G1 phase, which is representative of cells containing less DNA due to apoptotic DNA fragmentation from cellular endonucleases. At 24 hours post treatment, the sub-G1 phase cell population was significantly above the level observed for the untreated cells with an increase to $22.8 \%$ in 253JB-V cells and $5.0 \%$ in T24 cells. Figures $3 \mathrm{c}$ and $3 \mathrm{~d}$ show that the PAM treatment also induced cell cycle arrest. A significant increase in the percentage of 253JB-V and T24 cells in the quiescent G0 and intermediate $\mathrm{G} 1$ phase was maintained from 3 hours to 24 hours post treatment as compared to the untreated cells for both bladder cancer cell types. At 3 hours and 24 hours post treatment, a statistically lower percentage of the PAM treated cells are in the DNA synthesis $S$ phase as compared to the untreated cells for both cell types. The cell growth $\mathrm{G} 2$ phase and mitotic $\mathrm{M}$ phase for both untreated and treated cells were unaffected by PAM because of the fewer cells in the shorter G2 and $M$ phases. Overall, the results suggest that the cell cycle was arrested at G1. To validate this, expression of cyclin D, CDK 4 and p21 were investigated by western blot (Figure 3e). Cyclin D and CDK4 are important factors in driving the $S$ phase. Figure $3 e$ shows that both cyclin $D$ and CDK4 were down regulated. Conversely, p21 an inhibitor of CDK4, was up regulated.

\subsection{PAM induction of apoptosis of a bladder cancer tumour in vivo}

To investigate if the in vitro results are translatable in vivo, PAM was used to treat a subcutaneous 253JB$\checkmark$ bladder cancer tumour model in mice. In order to treat the subcutaneous tumour with PAM or untreated 
media for the control group, an incision was made into the mice skin to invert the subcutaneous tumour (connected to the skin) so that it was in direct contact with the PAM or untreated media (Figure 4a). At 24 hours post PAM treatment, the mice were sacrificed, and the region of the tumour was resected and analysed. Figure $4 \mathrm{~b}$ shows the tumour volume at 24 hours following treatment. The tumour volume was calculated to be $69.4 \pm 29.8 \mathrm{~mm}^{3}$ in the PAM treatment group and $115.7 \pm 13.2 \mathrm{~mm}^{3}$ in the untreated media control group. Figure $4 \mathrm{c}$ shows the tissue sections stained with: the terminal deoxynucleotidyl transferase dUTP nick end labelling (TUNEL) method for DNA fragmentation, a characteristic hallmark of apoptosis; anti-Ki-67 antibody to detect the presence of the Ki-67 protein, which is a cellular marker for proliferation; and hematoxylin and eosin (H\&E) to stain the cell nuclei, extracellular matrix and cytoplasm. The percentage of degenerated tumour tissue (indicated by the dashed lines in Figure 4c) was calculated by measuring the percentage of the tumour area containing TUNEL positive and KI-67 negative cells. The degenerated area of tumour tissue was calculated to be $51.4 \%$ in the PAM treatment group and $0 \%$ in untreated media control group (Figure $4 \mathrm{~d}$ ). The apoptosis index (Al) was determined by calculating the percentage of TUNEL positive cells from 10 randomly selected and anonymously scored high-power field images of the tissue sections. The Al was 2.8 in the PAM treatment group and 1.1 in the untreated media control group (Figure 4e). Overall, the in vivo results show that the PAM treatment increased apoptosis and decreased cell proliferation within the bladder cancer tumours and that this led to a decrease in the tumour volume 24 hours post treatment.

\section{Conclusion}

Bladder cancer is the fourth common cancer in the world. About $70 \%$ is non-muscle invasive bladder cancer (NMIBC) type. The standard treatment for NMIBC is endoscopic surgery by TURBT. Intravesical recurrence rate is very high after TURBT. Intravesical instillation immunotherapy by Bacillus CalmetteGuerin (BCG) is the most effective treatment post TURBT, but the treatment elicits multiple adverse sideeffects associated with a strong immune response and bacterial infections [36]. Overcoming these critical adverse reactions is of paramount importance to resolve for urologists. Therefore, in this study we investigated the possibility of low-temperature plasma activated solution (using PAM as a model solution), as a potential new intravesical chemotherapeutic approach for prophylactic treatment post TURBT.

The results presented in this study show that PAM treatment can induce death in bladder cancer cells through apoptosis. Apoptosis is a favourable form of cell death in cancer therapy as it reduces the chances of adverse side-effects resulting from inflammation and damage to surrounding healthy cells and tissue that can occur through other forms of cell death such as necrosis. This study shows that PAM induces elevated oxidative stress in the bladder cancer cells that initiates a cascade of intracellular signalling events leading to mitochondria dysfunction and cell cycle arrest, that in turn, triggers apoptosis in bladder cancer cells via the cytochrome c/caspase 3 pathway (Figure 5). Therefore, we conclude that an increase in mitochondrial ROS production and cell cycle arrest both contribute to apoptosis of the bladder cancer cells, which supports both the hypotheses proposed in the introduction section. The 
oxidative stress induced by PAM causes a disruption of mitochondrial function, which triggers the release cytochrome $\mathrm{C}$. The release of cytochrome $\mathrm{C}$ leads to a loss of mitochondrial membrane potential that increases mitochondrial ROS generation above the basal level. Both the release of cytochrome $\mathrm{C}$ and the generation of mitochondrial ROS activated by caspase 3 causes apoptosis. Caspase is one of the main factors associated with the apoptotic process. In the cell cycle, $\mathrm{G} 1 / \mathrm{S}$ phase is arrested in down regulation of cyclin D and CDK 4 and up regulation of p21. Both down regulated cyclin $D$ and CDK 4 do not allow the bladder cancer cells to move into the $\mathrm{S}$ phase leading to increased apoptosis.

In summary, this study highlights the possibility of using a plasma activated solution for prophylactic treatment of bladder cancer post-surgery. In a clinical setting, a plasma activated solution would be most likely be delivered into the bladder through a urethral catheter in the same way as BCG or an anticancer drug are delivered into the bladder. However, plasma activated solutions in bladder cancer treatment might help avoid common clinical problems associated with BCG such as atrophic bladder, infection, Reiter syndrome and interstitial pneumonia.

\section{Declarations}

\section{Acknowledgements}

This work supported by JSPS KAKENHI Numbers JP17K16798, JP19K18564, JP19K07483 and JP19K08280. EJS acknowledges the support from the Australian Research Council Future Fellowship FT190100263 and the National Health Medical Research Council Ideas Grant 2002510. JSO thanks support from Osaka City University Strategic Research Funds (Priority Research) in FY2019 and financial support by BioMedical Engineering Center (BMEC). This study was conceptualized by HF, JSO, KI, EJS, H.Furuta and AH. Methodology and investigations were performed by HF, JSO, KC, SY, AK, MF and MT. HF, EJS, JSO, HDL and RDS contributed to the writing.

\section{References}

1. M. G. Kong, G. Kroesen, G. Morfill, T. Nosenko, T. Shimizu, J. v. Dijk, and J. L. Zimmermann "Plasma medicine: an introductory review" New J. Phys.11:115012 2009.

2. E. A. Ratovitski, X. Cheng, D. Yan, J. H. Sherman, J. Canady, B. Trink, and M. Keidar "Anti-Cancer Therapies of 21st Century: Novel Approach to Treat Human Cancers Using Cold Atmospheric Plasma" Plasma Process. Polym.11:1128-1137 2014.

3. H. Tanaka, M. Mizuno, K. Ishikawa, K. Nakamura, H. Kajiyama, H. Kano, F. Kikkawa, and M. Hori "Plasma-Activated Medium Selectively Kills Glioblastoma Brain Tumor Cells by Down-Regulating a Survival Signaling Molecule, AKT Kinase" 1:265-277 2011.

4. H. Tanaka, M. Mizuno, K. Ishikawa, K. Nakamura, F. Utsumi, H. Kajiyama, H. Kano, S. Maruyama, F. Kikkawa, and M. Hori "Cell survival and proliferation signaling pathways are downregulated by plasma-activated medium in glioblastoma brain tumor cells" 2:207-220 2012. 
5. H. Tanaka, M. Mizuno, K. Ishikawa, H. Kondo, K. Takeda, H. Hashizume, K. Nakamura, F. Utsumi, H. Kajiyama, H. Kano, Y. Okazaki, S. Toyokuni, S. i. Akiyama, S. Maruyama, S. Yamada, Y. Kodera, H. Kaneko, H. Terasaki, H. Hara, T. Adachi, M. Iida, I. Yajima, M. Kato, F. Kikkawa, and M. Hori "Plasma with high electron density and plasma-activated medium for cancer treatment" Clinical Plasma Medicine3:72-76 2015.

6. F. Judée, C. Fongia, B. Ducommun, M. Yousfi, V. Lobjois, and N. Merbahi "Short and long time effects of low temperature Plasma Activated Media on 3D multicellular tumor spheroids" Scientific Reports6:21421 2016.

7. K. Nakamura, Y. Peng, F. Utsumi, H. Tanaka, M. Mizuno, S. Toyokuni, M. Hori, F. Kikkawa, and H. Kajiyama "Novel Intraperitoneal Treatment With Non-Thermal Plasma-Activated Medium Inhibits Metastatic Potential of Ovarian Cancer Cells" Scientific Reports7:6085 2017.

8. L. T.-H. Tan, K.-G. Chan, P. Pusparajah, W.-L. Lee, L.-H. Chuah, T. M. Khan, L.-H. Lee, and B.-H. Goh "Targeting Membrane Lipid a Potential Cancer Cure?" Frontiers in Pharmacology8: 2017.

9. W. J. van Blitterswijk, G. de Veer, J. H. Krol, and P. Emmelot "Comparative lipid analysis of purified plasma membranes and shed extracellular membrane vesicles from normal murine thymocytes and leukemic GRSL cells" Biochimica et Biophysica Acta (BBA) - Biomembranes688:495-504 1982.

10. N. Bernardes, and A. M. Fialho "Perturbing the Dynamics and Organization of Cell Membrane Components: A New Paradigm for Cancer-Targeted Therapies" International Journal of Molecular Sciences19:3871 2018.

11. M. C. Papadopoulos, and S. Saadoun "Key roles of aquaporins in tumor biology" Biochimica et Biophysica Acta (BBA) - Biomembranes1848:2576-2583 2015.

12. S. Saadoun, M. C. Papadopoulos, D. C. Davies, B. A. Bell, and S. Krishna "Increased aquaporin 1 water channel expression inhuman brain tumours" Br. J. Cancer87:621-623 2002.

13. D. Yan, A. Talbot, N. Nourmohammadi, J. H. Sherman, X. Cheng, and M. Keidar "Toward understanding the selective anticancer capacity of cold atmospheric plasma-A model based on aquaporins (Review)" Biointerphases10:040801 2015.

14. M. Keidar "Plasma for cancer treatment" Plasma Sources Sci. Technol.24:033001 2015.

15. J. Van der Paal, S.-H. Hong, M. Yusupov, N. Gaur, J.-S. Oh, R. D. Short, E. J. Szili, and A. Bogaerts "How membrane lipids influence plasma delivery of reactive oxygen species into cells and subsequent DNA damage: an experimental and computational study" PCCP21:19327-19341 2019.

16. M. Yusupov, A. Privat-Maldonado, R. M. Cordeiro, H. Verswyvel, P. Shaw, J. Razzokov, E. Smits, and A. Bogaerts "Oxidative damage to hyaluronan-CD44 interactions as an underlying mechanism of action of oxidative stress-inducing cancer therapy" Redox Biology43:101968 2021.

17. M. Keidar, A. Shashurin, O. Volotskova, M. A. Stepp, P. Srinivasan, A. Sandler, and B. Trink "Cold atmospheric plasma in cancer therapy" Physics of Plasmas20:057101-057108 2013.

18. R. L. Siegel, K. D. Miller, and A. Jemal "Cancer statistics, 2015" CA. Cancer J. Clin.65:5-29 2015.

19. J. Ferlay, E. Steliarova-Foucher, J. Lortet-Tieulent, S. Rosso, J. W. W. Coebergh, H. Comber, D. Forman, and F. Bray "Cancer incidence and mortality patterns in Europe: Estimates for 40 countries in 2012" 
Eur. J. Cancer49:1374-14032013.

20. K. A. Richards, N. D. Smith, and G. D. Steinberg "The Importance of Transurethral Resection of Bladder Tumor in the Management of Nonmuscle Invasive Bladder Cancer: A Systematic Review of Novel Technologies" The Journal of Urology191:1655-1664 2014.

21. H. M. Joh, S. J. Kim, T. H. Chung, and S. H. Leem "Reactive oxygen species-related plasma effects on the apoptosis of human bladder cancer cells in atmospheric pressure pulsed plasma jets" Appl. Phys. Lett.101:053703 2012.

22. S. Mohades, N. Barekzi, and M. Laroussi "Efficacy of Low Temperature Plasma against SCaBER Cancer Cells" Plasma Process. Polym.11:1150-1155 2014.

23. M. Keidar, R. Walk, A. Shashurin, P. Srinivasan, A. Sandler, S. Dasgupta, R. Ravi, R. Guerrero-Preston, and $\mathrm{B}$. Trink "Cold plasma selectivity and the possibility of a paradigm shift in cancer therapy" $\mathrm{Br} J$ Cancer105:1295-1301 2011.

24. J.-S. Oh, E. J. Szili, N. Gaur, S.-H. Hong, H. Furuta, R. D. Short, and A. Hatta "In-situ UV Absorption Spectroscopy for Monitoring Transport of Plasma Reactive Species through Agarose as Surrogate for Tissue " J. Photopolym. Sci. Technol.28:439-444 2015.

25. J.-S. Oh, E. J. Szili, N. Gaur, S.-H. Hong, H. Furuta, H, Kurita, A. Mizuno, A. Hatta, and R. D. Short "How to assess the plasma delivery of RONS into tissue fluid and tissue" J. Phys. D: Appl. Phys.49:304005 2016.

26. J.-S. Oh, M. Kakuta, H. Furuta, H. Akatsuka, and A. Hatta "Effect of plasma jet diameter on the efficiency of reactive oxygen and nitrogen species generation in water" Japanese Journal of Applied Physics55:06HD01 2016.

27. J.-S. Oh, E. J. Szili, A. Hatta, M. Ito, and T. Shirafuji "Tailoring the Chemistry of Plasma-Activated Water Using a DC-Pulse-Driven Non-Thermal Atmospheric-Pressure Helium Plasma Jet" Plasma2:127-137 2019.

28. X. Lu, G. V. Naidis, M. Laroussi, S. Reuter, D. B. Graves, and K. Ostrikov "Reactive species in nonequilibrium atmospheric-pressure plasmas: Generation, transport, and biological effects" Physics Reports630:1-84 2016.

29. B. Ghimire, E. J. Szili, P. Lamichhane, R. D. Short, J. S. Lim, P. Attri, K. Masur, K.-D. Weltmann, S.-H. Hong, and E. H. Choi "The role of UV photolysis and molecular transport in the generation of reactive species in a tissue model with a cold atmospheric pressure plasma jet" Appl. Phys. Lett.114:093701 2019.

30. A. G. Porter, and R. U. Jänicke "Emerging roles of caspase-3 in apoptosis" Cell Death Differ.6:99-104 1999.

31. Y.-L. P. Ow, D. R. Green, Z. Hao, and T. W. Mak "Cytochrome c: functions beyond respiration" Nature Reviews Molecular Cell Biology9:532-542 2008.

32. S. S. Korshunov, V. P. Skulachev, and A. A. Starkov "High protonic potential actuates a mechanism of production of reactive oxygen species in mitochondria" FEBS Letters416:15-18 1997. 
33. L. D. Zorova, V. A. Popkov, E. Y. Plotnikov, D. N. Silachev, I. B. Pevzner, S. S. Jankauskas, V. A. Babenko, S. D. Zorov, A. V. Balakireva, M. Juhaszova, S. J. Sollott, and D. B. Zorov "Mitochondrial membrane potential" Anal. Biochem.552:50-59 2018.

34. J. L. Dominguez-Escrig, J. D. Kelly, D. E. Neal, S. M. King, and B. R. Davies "Evaluation of the Therapeutic Potential of the Epidermal Growth Factor Receptor Tyrosine Kinase Inhibitor Gefitinib in Preclinical Models of Bladder Cancer" Clin. Cancer Res.10:4874-4884 2004.

35. B. Pucci, M. Kasten, and A. Giordano "Cell cycle and apoptosis" Neoplasia2:291-299 2000.

36. K. Decaestecker, and W. Oosterlinck "Managing the adverse events of intravesical bacillus CalmetteGuérin therapy" Res Rep UroП:157-163 2015.

\section{Figures}



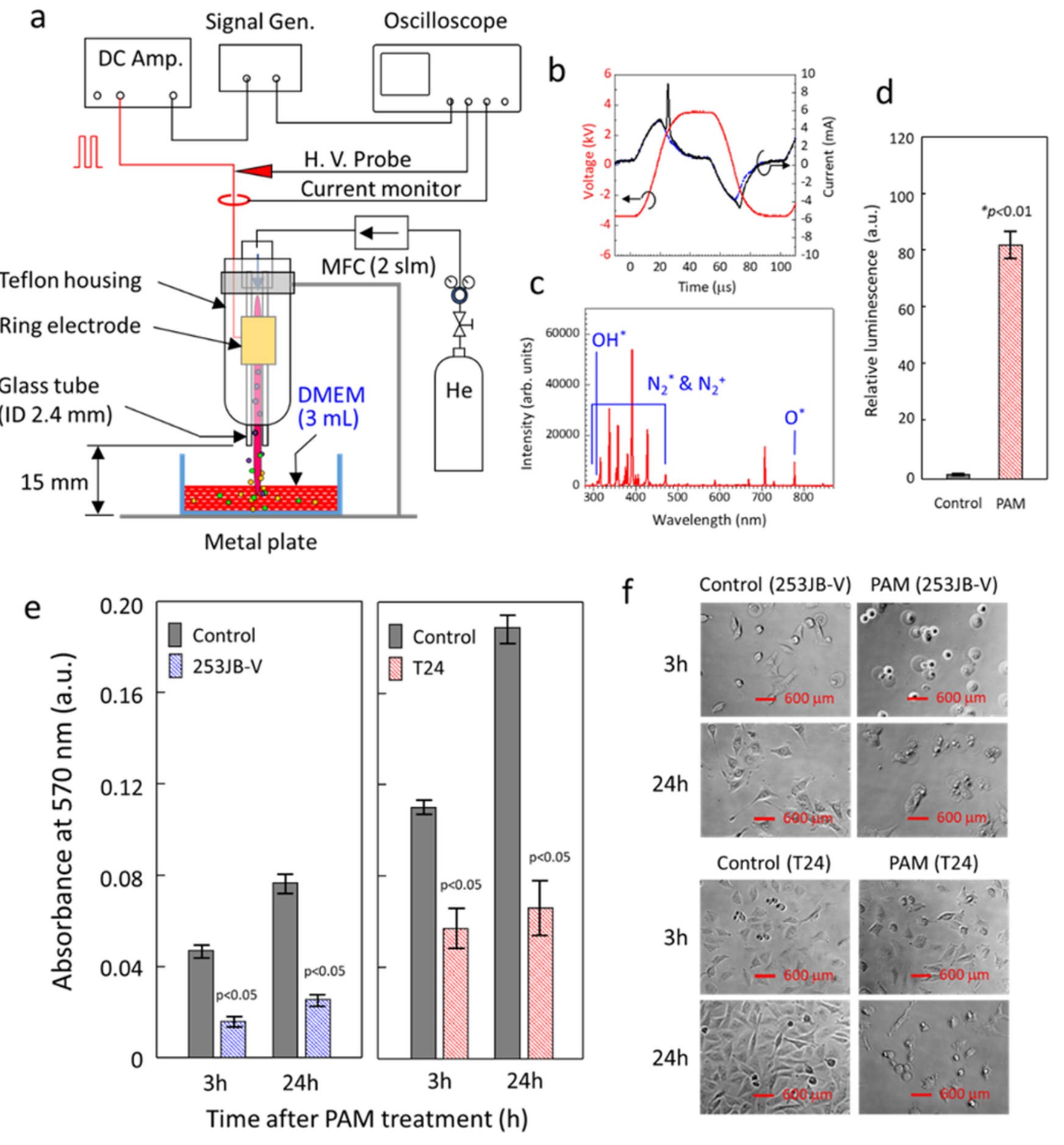

\section{Figure 1}

Overview of the procedure to prepare PAM for treatment of bladder cancer cells. (a) Set-up and procedure used to prepare PAM. (b) Electrical and (c) optical emission characteristics of the plasma jet. (d) Relative luminescence of luminol in PAM compared to untreated DMEM, which was used to compare the relative level of ROS. (e) Cell viability of cancer versus normal bladder following PAM treatment; Control = 
untreated cells measured at 24 hours after the PAM treatment. (f) Micrographs of the typical cell morphology before and after PAM treatment.
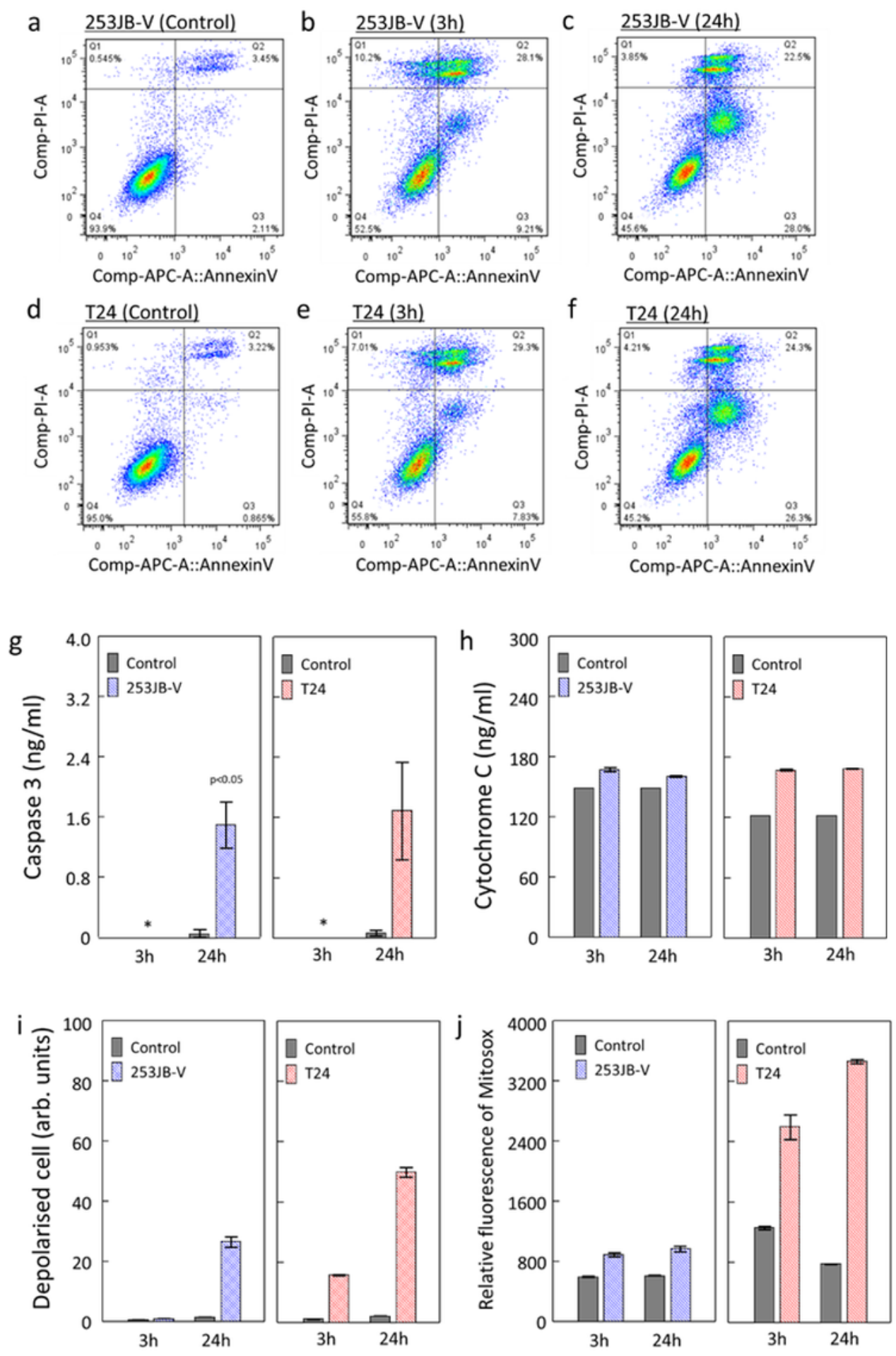

Figure 2

Mechanism of cell death induced by PAM treatment of bladder cancer cells. Flow cytometry graphs of 253JB-V bladder cancer cells stained with V-APC and PI (a) before PAM treatment (untreated control) and at (b) 3 hours and (c) 24 hours after PAM treatment; and T24 bladder cancer cells stained with V-APC and 
PI (d) before PAM treatment (untreated control) and at (e) 3 hours and (f) 24 hours after PAM treatment. Concentrations of $(\mathrm{g})$ caspase 3 (*data not available for the 3 hours timepoint subset) and $(\mathrm{h})$ cytochrome $\mathrm{c}$ in bladder cancer cells before (untreated control) and after PAM treatment. (i) Percentage of depolarised bladder cancer cells determined through measurement of the $\Delta \Psi_{\mathrm{m}}$ before (untreated control) and after PAM treatment. (j) Relative fluorescence of Mitosox to determine the relative level of mitochondrial ROS produced in bladder cancer cells.
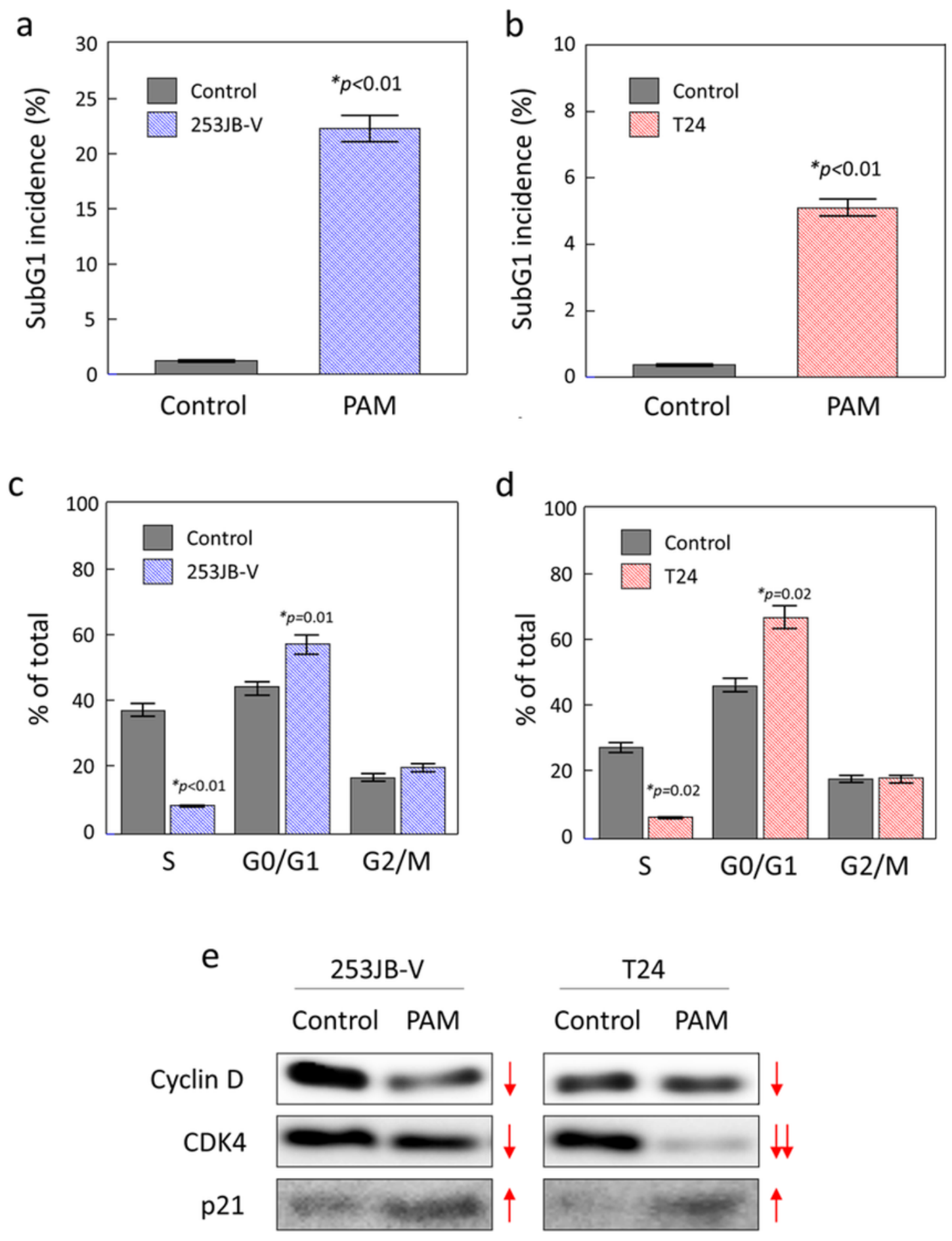

T24

Control PAM

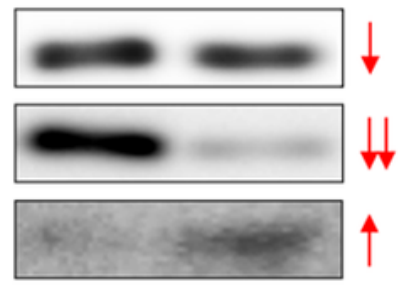

Figure 3 
Changes in the bladder cancer cell cycle induced by PAM. Incidence in the sub G1 phase for (a) 253JB-V and (b) T24 bladder cancer cells. Percentage of cell population in each stage of the cell cycle for (c) 253JB-V and (d) T24 bladder cancer cells. (e) Western blot analysis of cell cycle regulatory factors. Control in all figures refers to untreated cells.

a

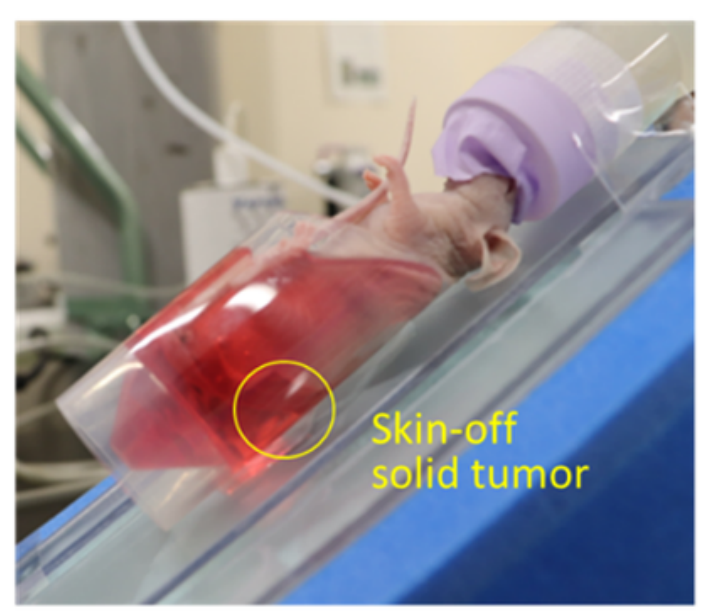

b

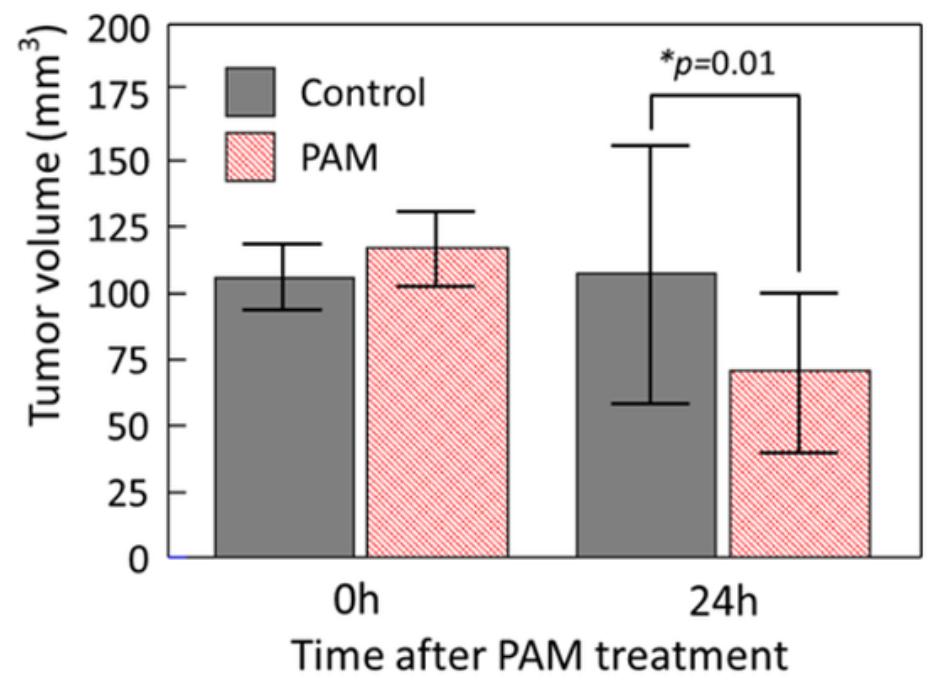

C
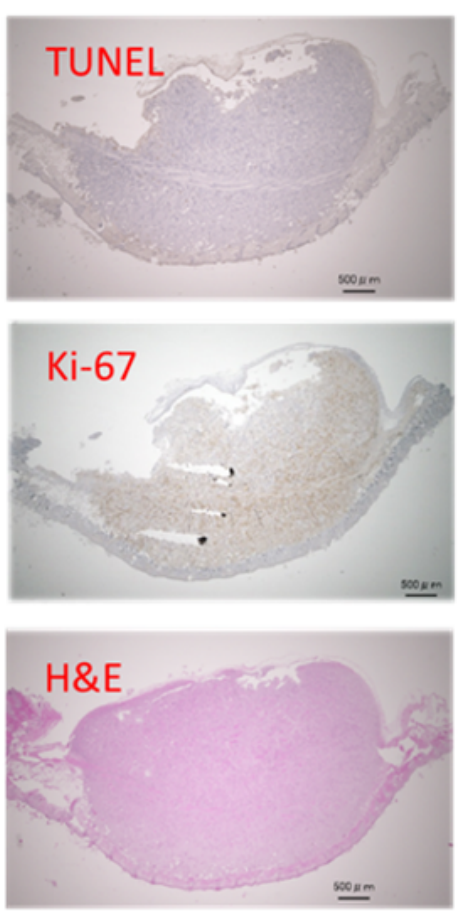

Control
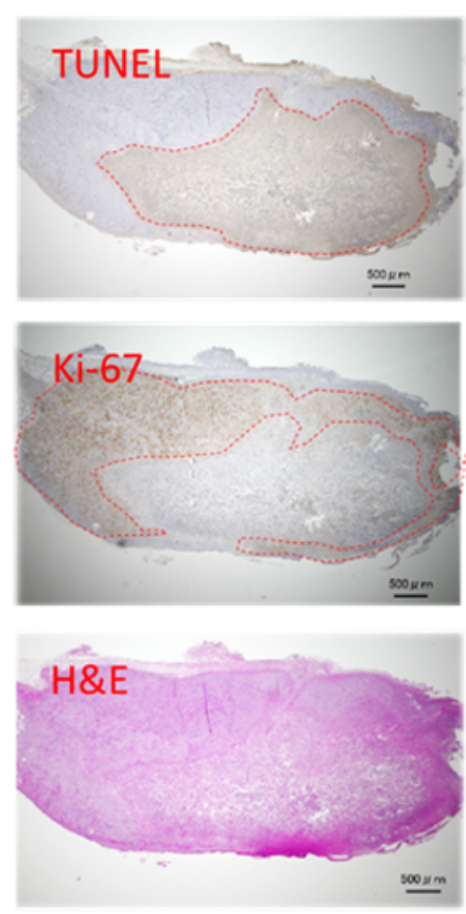

PAM

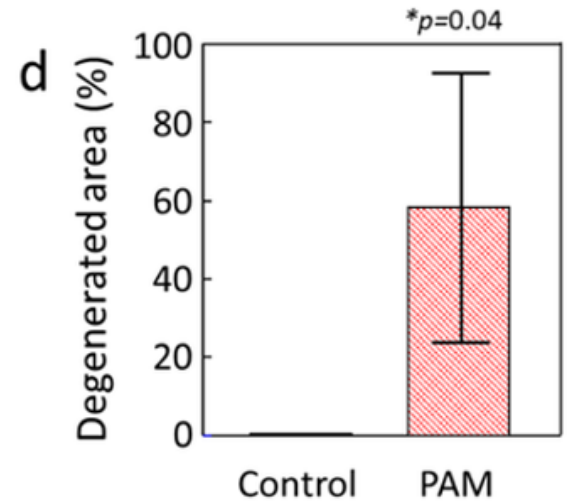

$\mathrm{e}$

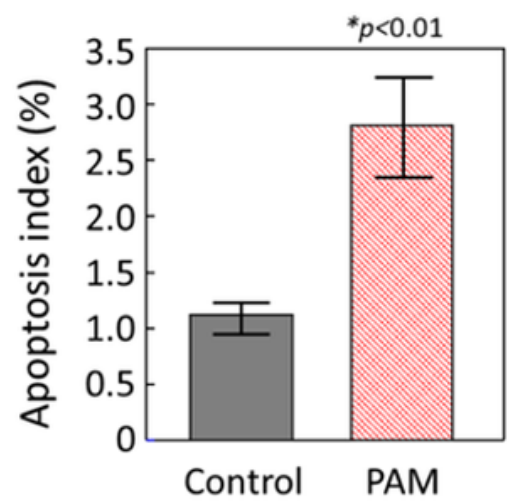

Figure 4 
Induction of apoptosis in a 3D cancer tumour by PAM. (a) Photograph of a mouse with a bladder cancer tumour immersed in a bath of PAM. (b) Graph of the tumour volume before and after PAM treatment. (c) Typical histology images of tumour sections stained with TUNEL, Ki-67 for untreated control tumour and PAM treated tumours. (d) Percentage of the degenerated tumour area. (e) Apoptosis index in the tumour. Control in all figures refers to untreated cells.

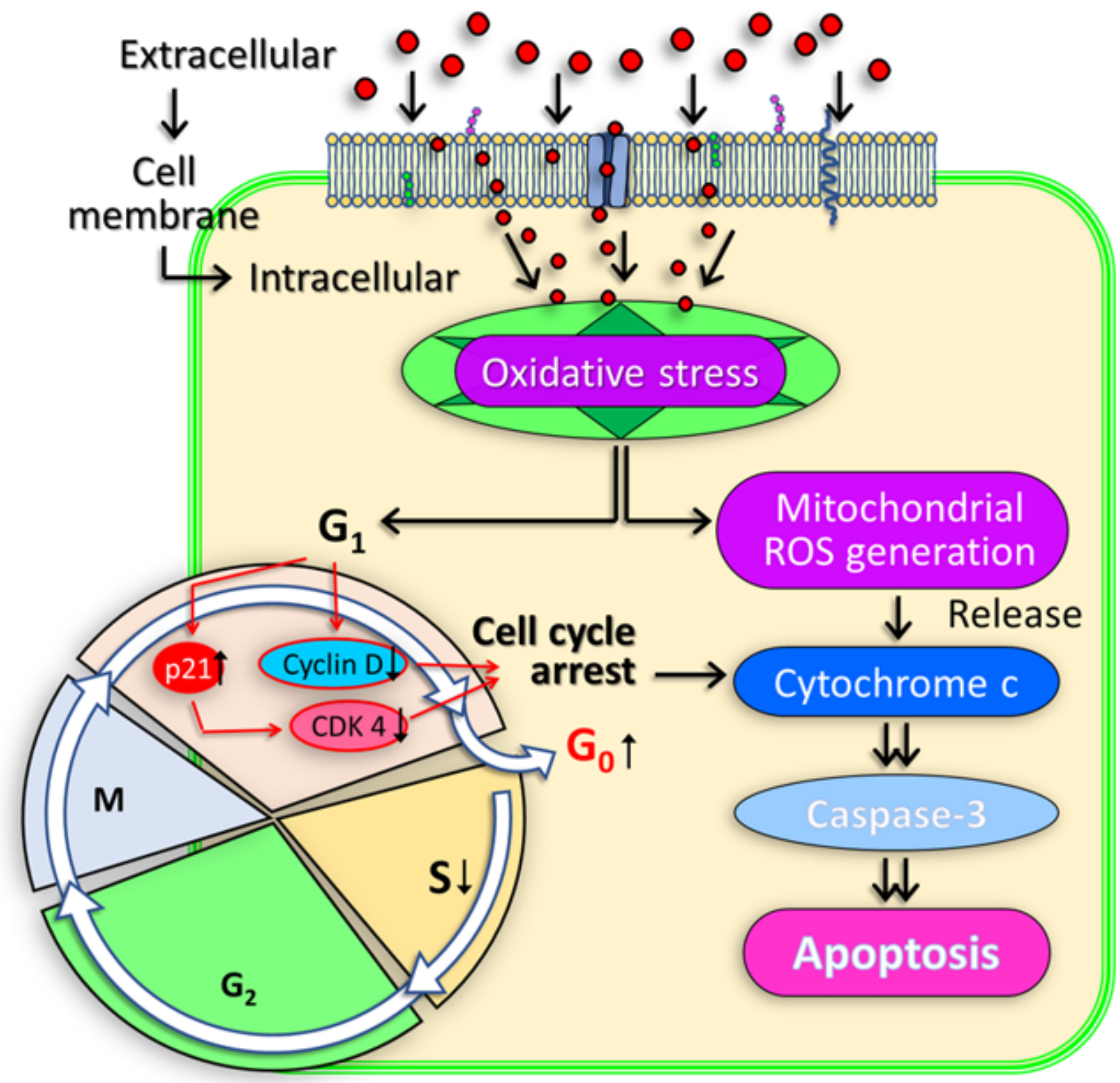

Figure 5

Overview of the signalling pathways involved in the PAM induction of apoptosis in bladder cancer cells. 